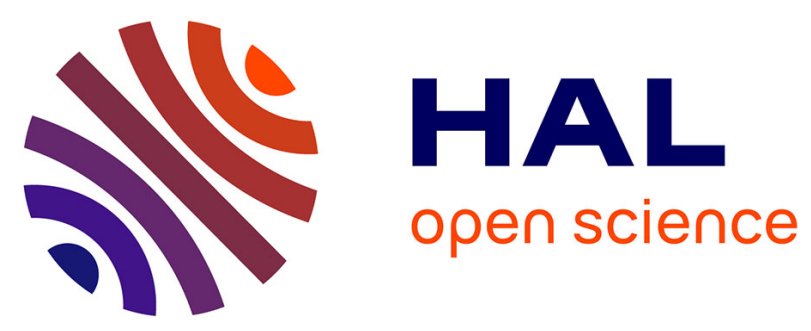

\title{
High-pressure transformations in liquid rubidium
}

Simon Ayrinhac, Victor Naden Robinson, Frédéric Decremps, Michel

Gauthier, Daniele Antonangeli, Sandro Scandolo, Marc Morand

\section{To cite this version:}

Simon Ayrinhac, Victor Naden Robinson, Frédéric Decremps, Michel Gauthier, Daniele Antonangeli, et al.. High-pressure transformations in liquid rubidium. Physical Review Materials, 2020, 4 (11), pp.113611. 10.1103/physrevmaterials.4.113611 . hal-03373682v2

\section{HAL Id: hal-03373682 \\ https://hal.science/hal-03373682v2}

Submitted on 11 Oct 2021

HAL is a multi-disciplinary open access archive for the deposit and dissemination of scientific research documents, whether they are published or not. The documents may come from teaching and research institutions in France or abroad, or from public or private research centers.
L'archive ouverte pluridisciplinaire HAL, est destinée au dépôt et à la diffusion de documents scientifiques de niveau recherche, publiés ou non, émanant des établissements d'enseignement et de recherche français ou étrangers, des laboratoires publics ou privés. 


\title{
High-pressure transformations in liquid rubidium
}

\author{
Simon Ayrinhac $\odot,{ }^{1}$ Victor Naden Robinson $\odot,{ }^{2}$ Frédéric Decremps $\odot,{ }^{1}$ Michel Gauthier $\odot,{ }^{1}$ \\ Daniele Antonangeli $\odot,{ }^{1}$ Sandro Scandolo $\odot,^{2}$ and Marc Morand ${ }^{1}{ }^{1}$ \\ ${ }^{1}$ Sorbonne Université, UMR CNRS 7590, Muséum National d'Histoire Naturelle, Institut de Minéralogie, \\ de Physique des Matériaux et de Cosmochimie, IMPMC, 75005 Paris, France \\ ${ }^{2}$ The “Abdus Salam” International Centre for Theoretical Physics, I-34151 Trieste, Italy
}

(Received 3 August 2020; accepted 23 October 2020; published 30 November 2020)

\begin{abstract}
An electronic-driven liquid-liquid phase transition in rubidium is revealed by picosecond acoustic measurements combined with $a b$ initio calculations. Picosecond acoustics were used to measure the melting line up to $10 \mathrm{GPa}$, finding the maximum in the melting curve at $7 \mathrm{GPa}$ and $555 \mathrm{~K}$. We observe the onset of a continuous liquid-liquid phase transition beginning around the melting maxima through until $16 \mathrm{GPa}$. Sound velocity shows a softening similar to that reported for liquid caesium, caused by a change in the bulk modulus during a crossover from the low-density to the high-density liquid. Guided by the ab initio calculations, we relate the changes in the thermoelastic properties to the progressive localization of the valence electrons in the pressure range of 6-16 GPa. At high pressure rubidium forms an electride liquid quantified by the appearance of interstitial quasiatoms localized in the valence electron density.
\end{abstract}

DOI: 10.1103/PhysRevMaterials.4.113611

\section{INTRODUCTION}

Alkali metals are considered archetypal simple metals at ambient conditions yet exhibit complex properties at high pressure: Phase diagrams possess melting curves with several maxima (Cs [1]) and host-guest (h-g) incommensurate solid phases [2-5]. Light alkali metals become liquid at ambient temperature on compression to very high pressure $(\mathrm{Li}$ at $50 \mathrm{GPa}$ [6], $\mathrm{Na}$ at $120 \mathrm{GPa}$ [5]) or present a metal-insulator transition (Na becomes transparent at $200 \mathrm{GPa}$ and ambient temperature [7]). Structural transformations in the liquid have been reported in $\mathrm{Cs}$ at $4 \mathrm{GPa}$ and $493 \mathrm{~K}[8,9]$ and in $\mathrm{Rb}$ between 7.5 and $12.9 \mathrm{GPa}$ at $573 \mathrm{~K}[10,11]$.

In parallel with the structural transformations, the electronic states of dense alkalis lose the free-electron metallic character in favor of orbital rehybridization and localization, leading to a reduction of metallicity and in the extreme case of $\mathrm{Na}$ even to the opening of a gap. Consensus is emerging, at least in light alkali metals, that electron localization is the result of the pressure-induced expulsion of valence electrons into the interstitial regions of the lattice leading to the formation of an electride state. The existence of electrides among solid alkali metals has been well established from pioneering $[12,13]$ and more recent work [3,14-16]. Light alkali metals were first recognized to be electride solids at high pressure: $\mathrm{Li}$ at $60 \mathrm{GPa}$ [16] and $\mathrm{Na}$ at $250 \mathrm{GPa}$ [12]. Alkali metals which form complex h-g structures $(\mathrm{Na}, \mathrm{K}, \mathrm{Rb})$ can be explained by their electride nature [3]. However, the effects are not as striking in heavy alkali metals. Moreover the presence of electrides remains to be confirmed and quantified for liquids.

At room temperature, solid $\mathrm{Rb}$ undergoes a series of transformations [17,18]: from bcc (Rb-I) to fcc (Rb-II) at $7 \mathrm{GPa}$
[19], to Rb-III (oC52) at $13 \mathrm{GPa}$ [20], to Rb-IV at $16 \mathrm{GPa}$ (incommensurate $\mathrm{h}-\mathrm{g}$ [21]) with an order-disorder transition [22] at $16.5 \mathrm{GPa}$, to $\mathrm{Rb}-\mathrm{V}$ above $20 \mathrm{GPa}(t \mathrm{I} 4)$ [19], and to $\mathrm{Rb}$-VI around $50 \mathrm{GPa}$ [23] (see Fig. 1). As is often the case, studies addressing the liquid are more controversial.

Early work interpreted anomalies of electrical conductivity and volume occurring on compression as signature of an electronic $s-d$ transition [24,25]. A first-order liquid-liquid phase transition was suggested by ab initio molecular dynamics (AIMD) at $12.9 \mathrm{GPa}$ and $573 \mathrm{~K}$ [10] on the basis of anomalies in the equation of state and the adiabatic coefficient, $\gamma$. Conversely, a gradual transition from a simple hard-sphere liquid to a complex liquid was recently proposed to occur in $\mathrm{Rb}$ at $7.5 \pm 1 \mathrm{GPa}$ [11] from measured x-ray diffraction (XRD). Measurements on heavy alkali metals are challenging due to their high reactivity, therefore thermodynamic data at high pressure $p$ and temperature $T$ is scarce or controversial (Cs $[8,26,27]$ and $\mathrm{Rb}[10,11,28])$.

Sound velocity measurements are a sensitive tool to detect structural transformations in the liquid state, such as Sn [29], Bi [30], Ga [31], or Cs [27]. Thus we perform picosecond acoustics (PA) measurements, a nondestructive technique well adapted to micrometric samples of liquid metals [32], inside a high-pressure diamond anvil cell (DAC). To investigate the microscopic origin of the anomalies observed by sound velocity measurements, AIMD simulations of the liquid were carried out, analyzing the structural and electronic changes. In this paper our combined experimental and computational study highlights an important structural transformation at $573 \mathrm{~K}$ from $6 \mathrm{GPa}$ and extending up to $14 \mathrm{GPa}$ resulting from the progressive change from the low-pressure free-electronlike liquid state to a high-pressure electride liquid. 


\section{METHODS}

Picosecond acoustics is a time-resolved pump-probe optical technique that generates propagating strain waves in solids or liquids [31,32]. An 800-nm infrared laser pump pulse (100 fs width, $80 \mathrm{MHz}$ repetition rate) is focused through a DAC at the surface of the metallic sample. The optically absorbed beam creates an acoustic strain pulse propagating across the sample, which is then detected at the opposite surface by a probe beam. The acoustic echoes induce changes in the refractive index of the liquid metal and displacement of the surface, which are measured by relative reflectivity variations seen with the probe beam. These surface reflections are used both to determine the travel time of the acoustic echoes, and hence sound velocity, and to identify melting as they strongly depend on the long-range order of the material [29,31] (see Supplemental Material [33] for details).

A sample chipped out from a Rb ingot (99.6\% purity Sigma Aldrich [33]) was loaded into a hole drilled in a rhenium gasket inside the DAC. To minimize chemical contamination and reaction with air, the loading was performed in a glove box $\left(<5 \mathrm{ppm} \mathrm{O}_{2}, \mathrm{H}_{2} \mathrm{O}\right)$. The pressure was measured by the shift of the temperature-independent fluorescence band of a $\mathrm{SrB}_{4} \mathrm{O}_{7}: 5 \% \mathrm{Sm}^{2+}$ gauge placed in the sample chamber to act as a pressure calibrant [34]. Pressure determination from the internal pressure gauge is compared to the estimation from the high-frequency edge of the diamond-anvil Raman signal measured at the center of the gasket hole [35].

Due to unknown stress distribution inside the diamond anvils, the P scale was corrected to known pressure points (ambient pressure if reached, for example). High temperature was generated by a calibrated resistive heater surrounding the DAC. The adiabatic sound velocity $v_{s}$ is obtained dividing the gasket thickness (around $20 \mu \mathrm{m}$ ) by the time of flight of the propagating acoustic strain wave. To ensure the hole thickness remained constant [36,37], measurements were made during sample decompression with a fine DAC membrane control $(0.05 \mathrm{bar} / \mathrm{min})$.

Density-functional theory calculations utilized the CASTEP code [38], using the generalized-gradient approximated exchange correlation [39], and a nine-electron ultrasoft pseudopotential with 2.1-bohr inner-core radius. A $300-\mathrm{eV}$ plane-wave cutoff was used in AIMD with $\mathrm{k}$ points sampled at the $\Gamma$ point. For solids, a grid density of $0.02 \AA^{-1}$ was used for structure optimization in agreement with Ref. [3]. Simulations ran at fixed density with a Nose-Hoover thermostat starting from a 128-atom melted bcc supercell quenched down to either 573 or $800 \mathrm{~K}$. The liquid was simulated up to $30 \mathrm{ps}$ along the 573 and $800 \mathrm{~K}$ isotherms with a 0.75 -fs time step and sampling the stress tensor every 10 steps.

After liquid equilibration, the mean internal energy, $U$, was used to determine isochoric specific heat, $C_{V}=\left(\frac{\partial U}{\partial T}\right)_{V}$. The isothermal compressibility, $\beta_{T}=\frac{1}{V}\left(\frac{\partial V}{\partial P}\right)_{T}$, where $V$ is the molar volume, was computed from the AIMD equation of state. The thermal expansion coefficient was calculated by $\alpha=\beta_{T}\left(\frac{\partial P}{\partial T}\right)_{V}$, and isobaric specific heat, $C_{p}$, was determined from Mayer's relation $C_{p}=C_{V}+V T \beta_{T}^{-1} \alpha^{2}$. From the adiabatic ratio, $\gamma=C_{p} / C_{V}$, the adiabatic sound velocity, $v_{S}$, can be computed with $v_{S}=\sqrt{\gamma / \rho \beta_{T}}$ where $\rho$ is the density.

\section{RESULTS}

The melting curve of $\mathrm{Rb}$ is well known below $7 \mathrm{GPa}$ [42-44] but remains debated at higher pressures. As illustrated in the phase diagram shown in Fig. 1 inconsistencies exist amongst available experimental data: The liquid-solid transition at $618 \mathrm{~K}$ from Lundegaard [22] appears at $17 \mathrm{GPa}$, as opposed to $24 \mathrm{GPa}$ by Gorelli et al. [11], yet both were determined by XRD. Here melting was detected using PA by imaging changes in wavefront patterns appearing at the sample surface [31]: Perfect circles indicate a liquid sample, whereas more complex patterns are observed in solid phases [33]. In addition, in liquid phase, the signal is less noisy or disturbed, and the position of the echo peak shifts due to variation of sound velocity between solid and liquid phases. Determination of the solid and melted states up to $10 \mathrm{GPa}$ confirm the first maximum at $7 \mathrm{GPa}$ and $555 \mathrm{~K}$ and are in good agreement with Kechin et al. [40] and Boehler and Zha [41].

The phase diagram and the pressure evolution of the liquid sound velocity (see Fig. 2) of $\mathrm{Rb}$ are both similar to that of Cs suggesting a double maximum in the melting line as already proposed by Boehler and Zha [41]. However, data beyond $10 \mathrm{GPa}$ are limited: The thesis of Lundegaard reports molten $\mathrm{Rb}$ at $3 p-T$ points [45], in overall agreement with the low-temperature melting line suggested by Boehler and Zha [41], in spite of possible problems of sample contamination occurring above $10 \mathrm{GPa}$. This thesis also contains unpublished liquid structure factors of $\mathrm{Rb}$ around 10,13,18 GPa [33].

In the liquid, we probed the adiabatic sound velocities along the $573 \mathrm{~K}$ isotherm, systematically observing in the multiple performed runs an anomalous behavior (Fig. 2).

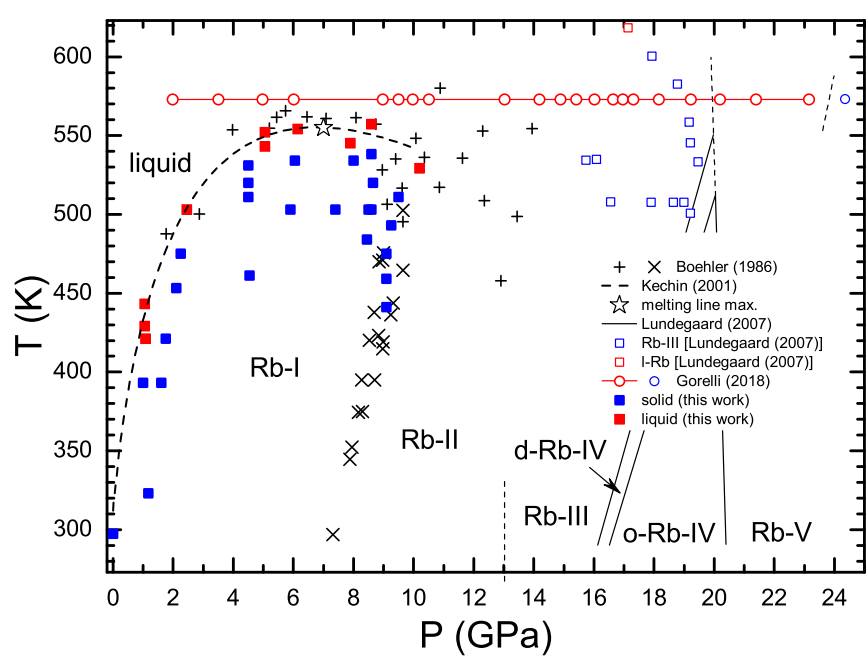

FIG. 1. Phase diagram of Rb: Solid (blue squares) and liquid (red squares) phases were detected using PA. The melting line is shown up to $10 \mathrm{GPa}$ (dashed line) according to the functional form given by Kechin [40]. The melting maximum is at $7 \mathrm{GPa}, 555 \mathrm{~K}$ (empty star). Transitions from bcc to fcc (crosses) and melting (pluses) are from Boehler [41]. Data at high $p-T[11,22,41]$ is scarce and not always consistent. Sound velocity measurements in this work were performed along the $573 \mathrm{~K}$ isotherm as in Gorelli et al. [11] (red circles). 


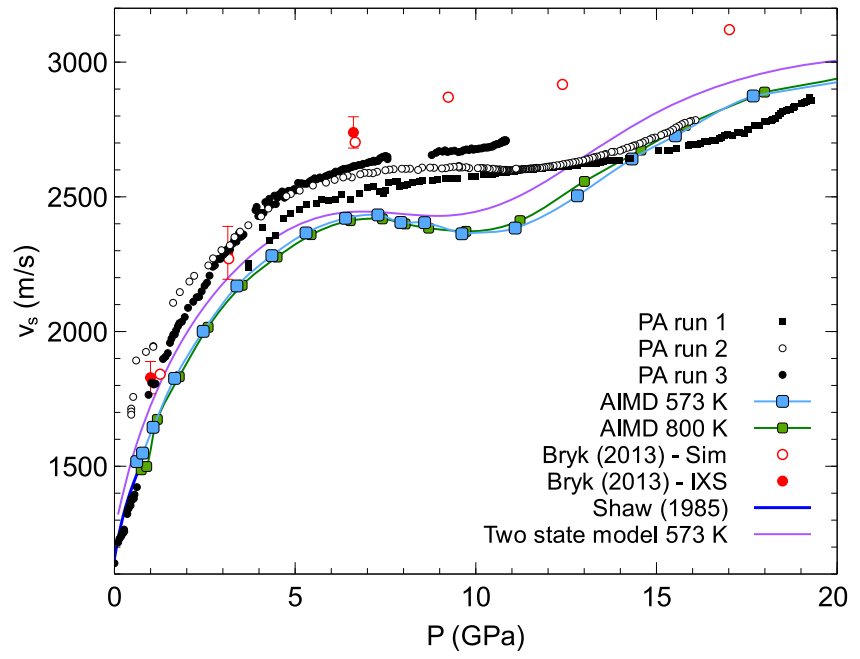

FIG. 2. Adiabatic sound velocities of liquid rubidium at $573 \mathrm{~K}$ as a function of pressure, measured by PA (black points), AIMD simulations (blue squares), and a two-state thermodynamic model (magenta) [33]. For comparison, ultrasonic measurements are shown from Shaw et al. [46] to $0.7 \mathrm{GPa}$ and the AIMD and IXS measurements from Bryk et al. [10].

Plateaus were found in $v_{s}$ between 6 and $14 \mathrm{GPa}$ in experimental runs 1 and 3, while run 2 and $\operatorname{AIMD}(573,800 \mathrm{~K}$ ) found an oscillation in this region similar to the $\mathrm{N}$-shape observed in liquid Cs [27]. A simple two-state model, which describes a system of entities in two thermodynamic states [47], in this case the two liquids, also finds an oscillation as the liquids mix. While PA measurements do not provide highly accurate absolute value of $v_{S}$ due to the uncertainty on sample thickness $\left(\Delta v_{S} \simeq \pm 100 \mathrm{~m} / \mathrm{s}\right)$, our results agree closely with the data of Shaw [46] to 0.7 GPa and inelastic X-ray scattering (IXS) determinations from Bryk [10] to $6 \mathrm{GPa}$. At pressures over $6 \mathrm{GPa}$ we find that PA and AIMD both have a lower $v_{s}$ than the simulations of Bryk, where PA data appears between the $v_{s}$ profiles of Bryk and AIMD performed in this work.

\section{DISCUSSION}

As is clear from the change in the Clapeyron slope, the liquid becomes denser than the solid after the first melting line maxima at about $7 \mathrm{GPa}$, and the $v_{s}$ plateau starts from this pressure. The complex, dense, phases of solid Rb (III, $\mathrm{IV}$, and $\mathrm{V}$ ) become stable around $14 \mathrm{GPa}$, pressure above which $v_{s}$ starts again to rise with increasing pressure. We thus interpret the anomalous pressure evolution of the adiabatic velocity as a direct consequence of a continuous but rapid transformation between two distinct microscopic states of the liquid. As shown in Fig. 3, while density does not show any sharp discontinuity in the transition region, where two states coexist, the bulk modulus shows a plateau, directly related to the plateau of the sound velocity. Conversely, sound velocity shows no evidence for anomalies due to a second transition expected at $12.9 \mathrm{GPa}$ according to Bryk [10].

Thermodynamic properties of liquid $\mathrm{Rb}$ are summarized in Fig. 3 which show no indications of a first- or secondorder phase transition, suggesting that this is a gradual
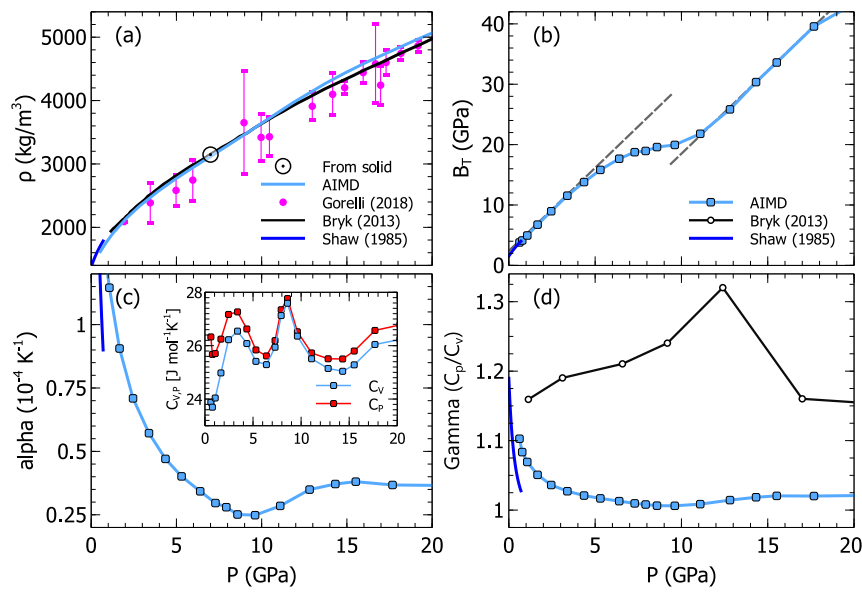

FIG. 3. Thermodynamic properties of liquid $\mathrm{Rb}$ at $573 \mathrm{~K}$ as a function of pressure including literature data from Gorelli [11] (XRD, pink), Bryk [10] (AIMD, black), and Shaw [46] (from ultrasonic measurements, blue). (a) Density; at the maxium of melting curve, the density of the solid (open dotted circle) is equal to that of the liquid. (b) Isothermal bulk modulus $B_{T}$ with dashed lines to guide the eye. (c) Thermal expansivity; inset: Specific heat capacities. (d) Adiabatic ratio $\gamma=C_{p} / C_{V}$.

crossover between two types of liquid. Density was obtained from AIMD simulations showing agreement with previous experimental and computational determinations [10,11]. A small inflection is visible around 8-9 GPa in correspondence to the anomalous sound velocity. The density of the bcc solid at $7 \mathrm{GPa}$, corresponding to the maximum in the melting curve, is $3140 \pm 20 \mathrm{~kg} / \mathrm{m}^{3}$ [33] in agreement with the density of the liquid as from AIMD and data of Bryk. The density of AIMD in this work is slightly lower than that of Bryk below $9 \mathrm{GPa}$ but significantly greater beyond $12 \mathrm{GPa}$. To $0.7 \mathrm{GPa}$ the density was calculated by a numerical procedure [31] from the accurate adiabatic sound velocity data of Shaw [46] extrapolated to $573 \mathrm{~K}$, consistent with AIMD. The isothermal bulk modulus, related to density by $B_{T}=$ $\rho(\partial p / \partial \rho)_{T}$, reveals a transition between two clear profiles as illustrated in Fig. 3(b). Thermal expansivity [Fig. 3(c)] exhibits a minima between 7 and $14 \mathrm{GPa}$ which is also expected by the two-state model, and the specific heat capacities appear to have local maxima and minima in this pressure region. The adiabatic ratio, Fig. 3(d), shows a decreasing trend with pressure similar to other compressed liquids $\left(\mathrm{H}_{2}\right.$ [48], $\mathrm{K}$ [49]), but in contrast to results from Bryk, who reported much higher values, and argued for a discontinuity at $12.9 \mathrm{GPa}$ not supported by our calculations. In fact, such high values of gamma would yield even greater sound velocities with a discontinuity if computed with the compressibility from AIMD in this work.

As is clear from the bulk modulus, liquid $\mathrm{Rb}$ is highly compressible at ambient conditions $\left(B_{0}=1.54 \mathrm{GPa}\right)$, with a $B_{0}$ value comparable with noble-gas solid $\mathrm{Ne}$ [50] $\left(B_{0}=\right.$ $1.07 \mathrm{GPa})$, and by $5 \mathrm{GPa} V / V_{0}=0.5$. At this compression ratio two $\mathrm{Rb}^{+}$ions are so close $(3.86 \AA)$ that the valence shell (radius of $2.44 \AA$ ) of one overlap with the impenetrable ion core of the nearest other (radius of $1.48 \AA$ ), pushing the conduction electrons to migrate inside voids between ion cores. An electronic localization starts to occur around $6 \mathrm{GPa}$ 


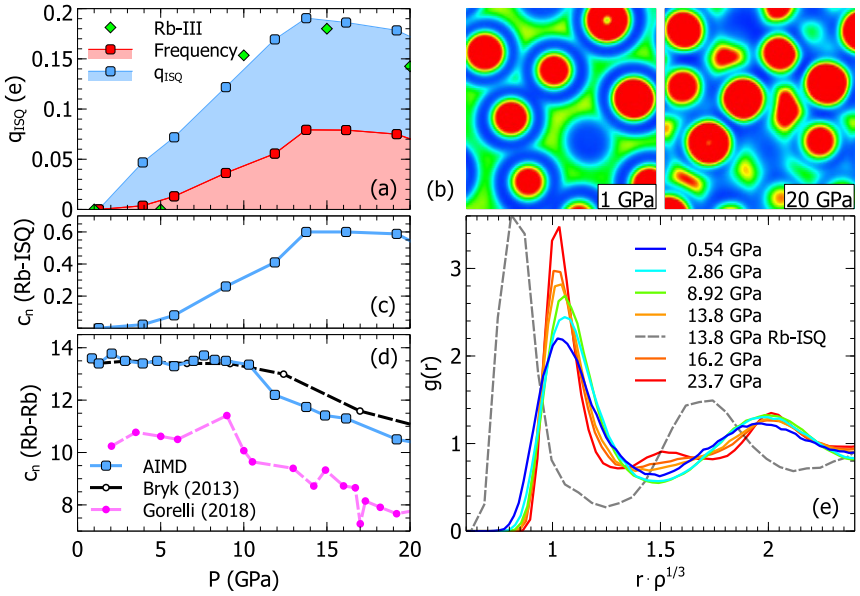

FIG. 4. Electronic and structural properties of liquid $\mathrm{Rb}$ as a function of pressure at $573 \mathrm{~K}$. (a) Average charge inside interstitial Bader maxima (blue) and their frequency (red) per $\mathrm{Rb}$ ion. (b) Electron localization function $(\max =0.70) 100$ cuts from AIMD snapshots at 1 and $20 \mathrm{GPa}$ showing localized interstitial charge (yellow to red amorphous shapes) appear between ions (red circles). (c) Coordination numbers for Rb ions to ISQs calculated by integrating PDFs up to the first minima and (d) for $\mathrm{Rb}-\mathrm{Rb}$ ions. (e) Normalized Rb-Rb PDFs at selected pressures showing the first minima evolve into a new maxima, and a Rb-ISQ PDF at $13.8 \mathrm{GPa}$ demonstrates the interstitial occupation of electride sites.

[see Fig. 4(a)], when a fraction of valence electrons start becoming confined in spaces between the ion cores, producing interstitial quasiatoms (ISQs) [16].

The localization is evident in AIMD snapshots comparing the interstitial electron localization at 1 and $20 \mathrm{GPa}$ in Fig. 4(b). ISQs were detected as off-atom maxima in a Bader analysis of the electron density [51] averaged over 100 snapshots at selected pressures [see Fig. 4(a)]. From 6 GPa, the number of ISQ (and their total charge) increase with pressure [see Fig. 4(a)] to $0.2 \mathrm{e} / \mathrm{ISQ}$ around $14 \mathrm{GPa}$, where $\mathrm{Rb}-\mathrm{III}$ and $\mathrm{Rb}$-IV become stable. Compared to liquid $\mathrm{Rb}$, in $\mathrm{Rb}-\mathrm{IV} 0.3 \mathrm{e} / \mathrm{ISQ}$ were found at $20 \mathrm{GPa}$ in the Bader scheme but around $1.1 \mathrm{e} / \mathrm{ISQ}$ when integrating electron localization function (ELF) basins [3], suggesting that the value in the liquid may be three times higher if ELF basins were integrated instead (to $0.6 \mathrm{e} / \mathrm{ISQ}$ at $14 \mathrm{GPa}$ ). The build up of electron localization with pressure was noted by Bryk [10] presenting three-dimensional ELF isosurfaces. A comparison with the liquid and solid phases of $\mathrm{Rb}$ to $50 \mathrm{GPa}$, and the distribution of charge within ISQs in the liquid, is shown in [33]. The Rb-Rb coordination number $c_{n}$ drops from $10 / 11 \mathrm{GPa}$ to around 8 [see Fig. 4(d)] which is when the ISQ's have significantly asserted their presence in the liquid. The decrease in $c_{n}$ is suggested, in analogy to the liquid-liquid transformation in Cs $[8,9]$, though the author stresses that higher-quality structural data are required to determine the nature of the high- and low-density liquids.

Miao et al. showed that for solids above $10 \mathrm{GPa}$ the "Rb 5s" valence orbital is favored to form an "ISQ 1s" state (see Fig. 5 of Ref. [14]). Here Rb is able to access these states already from $6 \mathrm{GPa}$ as the liquid is not restricted by symmetry. The creation of more ISQs, containing a fractional charge, attract
$\mathrm{Rb}^{+}$ions and offer additional screening of interactions which can reduce the internal energy and cause a deepening of the attractive part of the potential. This can cause the softening evidenced in both the isothermal bulk modulus and sound velocity.

Density-normalized pair distribution functions (PDFs) [Fig. 4(e)] show a simple Lennard-Jones liquid structure at low $\mathrm{P}$, and the apparition of an intermediate coordination shell between 8.9 and $13.8 \mathrm{GPa}$ in conjunction with the continuous decrease of coordination number similar to Cs [9]. The thesis of Lundegaard eluded the similar transformation in liquid Cs $[8,9]$ in the measured structure factor $S(q)$, suggesting a decrease in the coordination number $\left(c_{n}\right.$ drops to around 8), though the author stresses that higher-quality structural data are required to determine the nature of the high- and low-density liquids.

From 10/11 GPa, coordination number between $\mathrm{Rb}$ ions starts decreasing following the trend observed in solid phases: $c_{n}=14$ in Rb-I (bcc), 12 in Rb-II (fcc), 8/9 in RB-III (oc52), 9 in Rb-IV ( $h-g)$, and 8 in Rb-V (tI4). The coordination numbers measured by Gorelli [11] also decrease above $10 \mathrm{GPa}$ but are significantly lower than AIMD. Similarly, their PDFs [33] have much broader first peaks compared to AIMD results discussed here and of Bryk [10]. Lower coordination indicates an open structure, likely due to creation of directional bonds between $\mathrm{Rb}^{+}$cations and ISQ anions [15], creating a "complex" liquid, with a structure similar to the high-pressure solid phases mediated by the localized electrons.

\section{CONCLUSION}

In summary, we mapped the phase diagram of $\mathrm{Rb}$ at high-pressure probing melting and a liquid-liquid transition through sound velocity measurements and used $a b$ initio simulations to understand the nature of this transition. The $\mathrm{Rb}$ melting line was accurately measured to $10 \mathrm{GPa}$ by picosecond acoustic techniques, clearly showing a maximum at $7 \mathrm{GPa}$. Multiple experiments found a progressive pressureinduced transformation in liquid $\mathrm{Rb}$, highlighted by a plateau in the sound velocity between 6 and 14 GPa, while AIMD simulations similarly found an oscillation over the same pressure range. This anomaly is caused by an increase of compressibility concomitant to a continuous transition between two microscopically distinct states of the liquid. Absence of discontinuities in the density and compressibility clearly indicate that the phase transition is neither first nor second order, as claimed by previous work, but a crossover. The transition is related to a progressive localization of the electrons in interstitial regions as $\mathrm{Rb}$ forms a complex weak electride liquid. This is as well in stark contrast to previous explanations of $5 s$ electrons occupying the $4 d$ band, as partial density of states calculations can misinterpret electride materials, similarly shown for the solid alkali metals [3]. The maximum in the melting line requires the liquid to be less dense than the solid below $7 \mathrm{GPa}$ and denser than the solid above $7 \mathrm{GPa}$. Macroscopically the liquid continuously take on properties of the high-pressure phases (thermodynamically stable after the melting minima). The properties of the complex solid phases, including structural transformations and electride character, are mirrored in the liquid, as they are continuously adopted, 
with an extended transition region as the liquid-liquid transition mixes the two types of liquids.

\section{ACKNOWLEDGMENTS}

We thank H. Moutaabbid for his supporting help on the glove box and G. Le Marchand, Y. Guarnelli, and P.
Parisiadis for their support on high-pressure devices. D.A. has received funding from the European Research Council (ERC) under the European Union's Horizon 2020 research and innovation Programme (Grant No. 724690). Authors warmly thank Y. Garino, P. Rosier, and N. Dumesnil for their strong implication on the current picosecond acoustics set-up upgrade.
[1] A. Jayaraman, R. C. Newton, and J. M. McDonough, Phys. Rev. 159, 527 (1967).

[2] E. E. McBride, K. A. Munro, G. W. Stinton, R. J. Husband, R. Briggs, H.-P. Liermann, and M. I. McMahon, Phys. Rev. B 91, 144111 (2015).

[3] G. Woolman, V. Naden Robinson, M. Marqués, I. Loa, G. J. Ackland, and A. Hermann, Phys. Rev. Materials 2, 053604 (2018).

[4] V. N. Robinson, H. Zong, G. J. Ackland, G. Woolman, and A. Hermann, Proc. Natl. Acad. Sci. USA 116, 10297 (2019).

[5] E. Gregoryanz, L. F. Lundegaard, M. I. McMahon, C. Guillaume, R. J. Nelmes, and M. Mezouar, Science 320, 1054 (2008).

[6] C. L. Guillaume, E. Gregoryanz, O. Degtyareva, M. I. McMahon, M. Hanfland, S. Evans, M. Guthrie, S. V. Sinogeikin, and H. Mao, Nat. Phys. 7, 211 (2011).

[7] Y. Ma, M. Eremets, A. R. Oganov, Y. Xie, I. Trojan, S. Medvedev, A. O. Lyakhov, M. Valle, and V. Prakapenka, Nature 458, 182 (2009).

[8] S. Falconi, L. F. Lundegaard, C. Hejny, and M. I. McMahon, Phys. Rev. Lett. 94, 125507 (2005).

[9] S. Falconi and G. J. Ackland, Phys. Rev. B 73, 184204 (2006).

[10] T. Bryk, S. De Panfilis, F. A. Gorelli, E. Gregoryanz, M. Krisch, G. Ruocco, M. Santoro, T. Scopigno, and A. P. Seitsonen, Phys. Rev. Lett. 111, 077801 (2013).

[11] F. A. Gorelli, S. De Panfilis, T. Bryk, L. Ulivi, G. Garbarino, P. Parisiades, and M. Santoro, J. Phys. Chem. Lett. 9, 2909 (2018).

[12] J. B. Neaton and N. W. Ashcroft, Phys. Rev. Lett. 86, 2830 (2001).

[13] B. Rousseau and N. W. Ashcroft, Phys. Rev. Lett. 101, 046407 (2008).

[14] M.-S. Miao and R. Hoffmann, Acc. Chem. Res. 47, 1311 (2014).

[15] M.-s. Miao and R. Hoffmann, J. Am. Chem. Soc. 137, 3631 (2015).

[16] M.-S. Miao, R. Hoffmann, J. Botana, I. I. Naumov, and R. J. Hemley, Angew. Chem. 129, 992 (2017).

[17] H. Olijnyk and W. Holzapfel, Phys. Lett. A 99, 381 (1983).

[18] M. Winzenick, V. Vijayakumar, and W. B. Holzapfel, Phys. Rev. B 50, 12381 (1994)

[19] V. F. Degtyareva, Solid State Sci. 36, 62 (2014).

[20] R. J. Nelmes, M. I. McMahon, J. S. Loveday, and S. Rekhi, Phys. Rev. Lett. 88, 155503 (2002).

[21] U. Schwarz, A. Grzechnik, K. Syassen, I. Loa, and M. Hanfland, Phys. Rev. Lett. 83, 4085 (1999).

[22] L. F. Lundegaard, High-pressure diffraction studies of rubidium phase IV, Ph.D. thesis, University of Edinburgh, 2007.
[23] U. Schwarz, K. Syassen, A. Grzechnik, and M. Hanfland, Solid State Commun. 112, 319 (1999).

[24] S. M. Stishov and I. N. Makarenko, Zh. Eksp. Teor. Fiz. 54, 708 (1968) [Sov. Phys. JETP 27, 378 (1968).

[25] I. N. Makarenko, V. A. Ivanov, and S. M. Stishov, JETP Lett. 18, 187 (1973).

[26] T. Hattori, Phys. Rev. B 97, 100101(R) (2018).

[27] F. Decremps, S. Ayrinhac, M. Gauthier, D. Antonangeli, M. Morand, Y. Garino, and P. Parisiades, Phys. Rev. B 98, 184103 (2018).

[28] I. Loa, L. F. Lundegaard, M. I. McMahon, S. R. Evans, A. Bossak, and M. Krisch, Phys. Rev. Lett. 99, 035501 (2007).

[29] L. Xu, Y. Bi, X. Li, Y. Wang, X. Cao, L. Cai, Z. Wang, and C. Meng, J. Appl. Phys. 115, 164903 (2014).

[30] M. Emuna, M. Mayo, Y. Greenberg, E. Caspi, B. Beuneu, E. Yahel, and G. Makov, J. Chem. Phys. 140, 094502 (2014).

[31] S. Ayrinhac, M. Gauthier, G. L. Marchand, M. Morand, F. Bergame, and F. Decremps, J. Phys.: Condens. Matter 27, 275103 (2015).

[32] F. Decremps, M. Gauthier, S. Ayrinhac, L. Bove, L. Belliard, B. Perrin, M. Morand, G. Le Marchand, F. Bergame, and J. Philippe, Ultrasonics 56, 129 (2015).

[33] See Supplemental Material at http://link.aps.org/supplemental/ 10.1103/PhysRevMaterials.4.113611 for details on the experimental data analysis (Secs. 1-2), the two-state thermodynamic model (Sec. 3), the thermodynamic parameters (Sec. 5), and the data obtained by AIMD simulations [structure factors and pair distribution functions (Sec. 4), interstitial quasi-atoms (Sec. 6), equation of state and diffusion coefficient (Sec. 7)].

[34] F. Datchi, A. Dewaele, P. Loubeyre, R. Letoullec, Y. Le Godec, and B. Canny, High Press. Res. 27, 447 (2007).

[35] S. Ono, K. Mibe, and Y. Ohishi, J. Appl. Phys. 116, 053517 (2014).

[36] A. Dewaele, J. H. Eggert, P. Loubeyre, and R. Le Toullec, Phys. Rev. B 67, 094112 (2003).

[37] S. Ayrinhac, M. Gauthier, L. E. Bove, M. Morand, G. L. Marchand, F. Bergame, J. Philippe, and F. Decremps, J. Chem. Phys. 140, 244201 (2014).

[38] S. J. Clark, M. D. Segall, C. J. Pickard, P. J. Hasnip, M. I. J. Probert, K. Refson, and M. C. Payne, Z. Kristallogr. 220, 567 (2005).

[39] J. P. Perdew, K. Burke, and M. Ernzerhof, Phys. Rev. Lett. 77, 3865 (1996).

[40] V. V. Kechin, Phys. Rev. B 65, 052102 (2001).

[41] R. Boehler and C.-S. Zha, Physica B+ C 139, 233 (1986).

[42] H. Luedemann and G. Kennedy, J. Geophys. Res. 73, 2795 (1968).

[43] A. Nikolaenko, I. Makarenko, and S. Stishov, Solid State Commun. 27, 475 (1978). 
[44] R. Boehler and D. Young, J. Non-Cryst. Solids 61, 141 (1984). [45] L. F. Lundegaard, Ph.D. thesis, University of Edinburgh, 2007. [46] G. H. Shaw and D. A. Caldwell, Phys. Rev. B 32, 7937 (1985). [47] V. Holten and M. A. Anisimov, Sci. Rep. 2, 713 (2012).

[48] K. Matsuishi, E. Gregoryanz, H.-k. Mao, and R. J. Hemley, J. Chem. Phys. 118, 10683 (2003).
[49] H. Li, H. Ding, Y. Tian, Y. L. Sun, and M. Li, AIP Adv. 9, 075018 (2019).

[50] A. Dewaele, F. Datchi, P. Loubeyre, and M. Mezouar, Phys. Rev. B 77, 094106 (2008).

[51] S. G. Dale and E. R. Johnson, J. Phys. Chem. A 122, 9371 (2018). 